Research Article

\title{
Chitosan-Capped Au Nanoparticles for Laser Photothermal Ablation Therapy: UV-Vis Characterization and Optothermal Performances
}

\author{
Giancarlo Margheri ${ }^{(D},{ }^{1}$ Silvana Trigari, ${ }^{1}$ Mariabeatrice Berti, ${ }^{2}$ Maurizio Muniz Miranda, ${ }^{1}$ \\ and Rita Traversi $\left(\mathbb{D}^{2}\right.$ \\ ${ }^{1}$ Institute for Complex Systems, National Research Council, Via Madonna del Piano 10, 50019 Sesto Fiorentino, Firenze, Italy \\ ${ }^{2}$ Department of Chemistry "Ugo Schiff”, University of Florence, Via della Lastruccia 13, 50019 Sesto Fiorentino, Firenze, Italy \\ Correspondence should be addressed to Giancarlo Margheri; giancarlo.margheri@cnr.it
}

Received 31 January 2018; Accepted 12 April 2018; Published 10 July 2018

Academic Editor: Artem E. Masunov

Copyright (c) 2018 Giancarlo Margheri et al. This is an open access article distributed under the Creative Commons Attribution License, which permits unrestricted use, distribution, and reproduction in any medium, provided the original work is properly cited.

\begin{abstract}
We have reported on the synthesis and characterization of near-infrared- (NIR-) absorbing colloidal nanoparticles prepared by exploiting the one-step reaction of $\mathrm{HAuCl}_{4}$ and $\mathrm{Na}_{2} \mathrm{~S}_{2} \mathrm{O}_{3}$, followed by their stabilization with chitosan. This reaction also produces a big amount of unwanted nanoparticles detuned with respect to the NIR spectral region. For this reason, it is usually assumed that the product has to be filtered and enriched to enhance its NIR absorption, and the possible exploitation of the simpler raw product has never been worthy to be considered. Aiming to investigate this missing aspect, we chose to avoid the purification steps and rather focused on the preparation of the unrefined colloid, identifying the synthesis conditions that maximize its NIR absorbance and, subsequently, testing it as an optothermal transducer by measuring its molar heating rate (MHR). As expected, we found that the performances of the raw colloid are indeed lower than those of its refined version, but only to a limited extent. Moreover, MHR is unexpectedly higher than that deducible for other classical NIR-absorbing nanoparticles, like Au nanorods or Au nanostars. Thus, the product of the simpler preparation protocol appears as a competitive trade-off solution between easy manufacturing and optothermal performances.
\end{abstract}

\section{Introduction}

Laser photothermal ablation assisted by plasmonic nanoparticles has emerged as one of the most promising approaches to defeat cancer diseases. A large amount of work has been produced in this field, considering the various crucial aspects for the assessment of a protocol ensuring the effectiveness of therapy, safety, and optimal tumor targeting at the same time. While the specificity of tumor targeting is generally enhanced by proper particle decoration [1-3] or by the manufacturing of specific biological vectors doped with $\mathrm{Au}$ nanoparticles (AuNPs) [4-7], biocompatibility is ensured by decoration with nontoxic moieties [6, 7]. Effectiveness of light/heat conversion is mainly related to the shape and size of nanoparticles or their agglomerates. In fact, such features manage the absorption efficiency of laser radiation and of heat delivery to the surrounding media with benefits exploited for instance in bio-oriented and sensing applications. In order to obtain maximum penetration in biological tissues, infrared radiation has to be used to stimulate AuNPs and this restricts the choice to particles which underwent uneven growth. As of today, the current literature is rich in examples: nanorods, nanostars, nanocages, nanohexapods [8], nanoprisms [9], or even bio-induced AuNP clusters [10], that absorb fairly well in the near-infrared (NIR) region of the electromagnetic spectrum. Nonspherical growth is usually obtained via chemical routes using a highly toxic surfactant, namely, cetyltrimethylammonium bromide (CTAB), which must be mandatorily removed after the synthesis. Moreover, in order to keep colloidal nanoparticles stable, a new nontoxic surfactant must be added to the nanoparticle surface, for instance, polyethylene glycol (PEG), one of the most commonly used 
compound. The process thus requires multiple steps, and synthesis yield is an important concern. To overcome these problems, nanoparticles synthesized without CTAB and based on the reaction between $\mathrm{HAuCl}_{4}$ and sodium thiosulfate $\left(\mathrm{Na}_{2} \mathrm{~S}_{2} \mathrm{O}_{3}\right)$ [11-13] using chitosan as stabilizer [14] have been taken into consideration. Chitosan is a biobased polysaccharide, derived from shrimps and crab shells. It is nontoxic, biodegradable, biocompatible, and easily processable $[15,16]$.

Margheri et al. just demonstrated the efficiency of this reaction's product [7] in tumor treatment and cell therapy. However, no systematic study was performed to assess the characteristic features of the colloid, for instance, the molar ratio of the two reagents needed to obtain the highest NIR absorption.

This reaction, derived from that proposed for the first time by Zhou et al. [17], between tetrachloroauric acid and sodium sulfite is, by itself, effective to generate NIRabsorbing AuNPs. Unfortunately, it can also follow another pathway, whose result is a large amount of small spheroidal nanoparticles that cannot be used to absorb NIR radiation. Moreover, in clinically oriented applications, they would only increase off-target accumulation in the reticuloendothelial system (RES) organs, whose cells are furthermore expected to strongly interact with the positively charged chitosan-capped nanoparticles, unless they are safely carried by proper shielding cell vectors [7].

The natural way to overcome the presence of non-NIR nanoparticles and increase NIR efficiency is to filter them out and obtain a refined colloidal solution of concentrated NIRabsorbing nanoparticles. Following this path, efficient colloids capable of reaching hyperthermic regimes in few minutes at low laser intensities have been obtained. However, the refinement of the raw colloidal solution is not trivial as it suffers from typical issues induced by standard manufacturing steps (centrifugation and filtration) necessary to obtain NIR colloid with an enhanced content of NIRresonating AuNPs. The question arises whether the raw colloidal suspension shows sufficiently high thermal performances to counterbalance the drawbacks of further refinement stages without any further handling.

In this paper, we report on our recent spectroscopic and thermal characterization of chitosan-stabilized colloids, based on $\mathrm{HAuCl}_{4} / \mathrm{Na}_{2} \mathrm{~S}_{2} \mathrm{O}_{3}$ reduction synthesis, that did not undergo further treatments. First, we identified the necessary molar ratio of $\mathrm{HAuCl}_{4}$ and $\mathrm{Na}_{2} \mathrm{~S}_{2} \mathrm{O}_{3}$ to produce the highest absorbance around $810 \mathrm{~nm}$ wavelength. Using this ratio, we checked the optothermal performances of the product and found that, as expected, they are lower than the ones of the refined product, but only up to a moderate extent. Thus, our product seems to be highly competitive when pointing at applications specifically aimed at mass production, where the cited purification problems can constitute a major concern.

\section{Materials and Methods}

$\mathrm{HAuCl}_{4}$ (tetrachloroauric(III) acid) and $\mathrm{Na}_{2} \mathrm{~S}_{2} \mathrm{O}_{3}\left(5 \mathrm{H}_{2} \mathrm{O}\right)$ (sodium thiosulfate) were purchased from Sigma-Aldrich; ultrapure Milli-Q water with resistivity $18 \mathrm{M} \Omega \cdot \mathrm{cm}$ was used as the solvent; high molecular weight chitosan, with molecular weight of $106 \mathrm{kDa}$ and deacetylation degree of $79 \%$, was purchased from Heppe Medical. These reagents were then used to prepare the solutions. All of them, including the diluted ones, were preserved at low temperature $\left(4^{\circ} \mathrm{C}\right.$, fridge temperature). Dark glass flasks are used to contain these solutions to avoid direct exposure to light.

The UV-Vis analysis of the produced colloids was performed with either one of two different instruments, namely, AvaSpec-3648 spectrometer, using a quartz cuvette with optical path (OP) of $2 \mathrm{~mm}$, used to record the spectra shown in Figure 4, and an Agilent Cary 60 UV-Vis, using a quartz cuvette with OP of $1 \mathrm{~mm}$, for all the other characterizations.

The morphology and dimensions of the produced AuNPs were examined with transmission electron microscopy (TEM) using a Philips CM12 cryo-Gatan UHRST 500 @ $100 \mathrm{kV}$ transmission electronic microscope.

The stabilization of the colloidal solutions was checked by measuring their $\zeta$-potential, using a Zetasizer Nano ZS90 instrument (Malvern Instruments).

Optothermal tests were performed by irradiating the colloidal solution with a Roithner Lasertechnik diode laser module (808 nm wavelength; $0.5 \mathrm{~W}$ nominal CW output power).

The temperature raise was measured with a FLIR thermal camera and B335 (spectral range $7.5-13 \mu \mathrm{m}$; sensitivity: $50 \mathrm{mK}$ ) equipped with a $18 \mathrm{~mm}$ focal length objective and a field of view (FOV) of $25^{\circ} \times 19^{\circ}$. AuNPs were prepared by mixing fixed volumes of $\mathrm{HAuCl}_{4} \cdot 15.1 \mathrm{mM}(0.3 \mathrm{~mL})$ and Milli$\mathrm{Q}$ water $(2.2 \mathrm{~mL})$ in order to have an $\mathrm{HAuCl}_{4}$ final concentration of $1.7 \mathrm{mM}$. Variable volumes of $\mathrm{Na}_{2} \mathrm{~S}_{2} \mathrm{O}_{3} \cdot 3 \mathrm{mM}$ in $\mathrm{mQ}$ water were then rapidly added, and the samples were vortexed for $20 \mathrm{~s}$. Within the first minute after the addition of $\mathrm{Na}_{2} \mathrm{~S}_{2} \mathrm{O}_{3}$ to $\mathrm{HAuCl}_{4}$, samples developed a brownish color that rapidly turned to bluish purple.

It is worth to notice that the rate of color changing was found to be temperature dependent; in the case of lower ambient temperature such as $17^{\circ} \mathrm{C}$, the development of brownish color was reached after about 10 minutes. This fact suggested the reaction to be slower at lower temperature so that a larger time interval (about 1:20-1:30 h) was needed to consider the reaction completed and before proceeding with any other treatment, such as the addition of chitosan.

Samples were then set to rest for 1 hour, at ambient temperature $\left(24-26^{\circ} \mathrm{C}\right)$. After this time, the particles can be stabilized by adding a chitosan solution in $\mathrm{CH}_{3} \mathrm{COOH}$ $\left(\mathrm{HAuCl}_{4} /\right.$ chitosan molar ratio $\left.=5\right)$ and gently shaking the solutions on a magnetic stirrer for about $8 \mathrm{~h}$. Different molar ratios, $R=\left[\mathrm{HAuCl}_{4}\right] /\left[\mathrm{Na}_{2} \mathrm{~S}_{2} \mathrm{O}_{3}\right]$, were tested in order to check the tunability of the absorption in the infrared as reported by [11] and in particular towards $1064 \mathrm{~nm}$, where an other biological transparency window used for the applications of the Nd:YAG laser sources is present. Molar ratios $R$ spanning from 1.7 to 3.5 were chosen to this purpose. Optothermal tests on the colloid were performed considering $R=2.74$.

One sample (concentration of Au mass $=1.45 \mathrm{mM}$ and $\mathrm{OD}=6$, corresponding to an optical path of $10 \mathrm{~mm}$ ), obtained with the standard synthesis procedure was injected in a circular hole carved in a PTFE block (hole diameter: $8 \mathrm{~mm}$; 


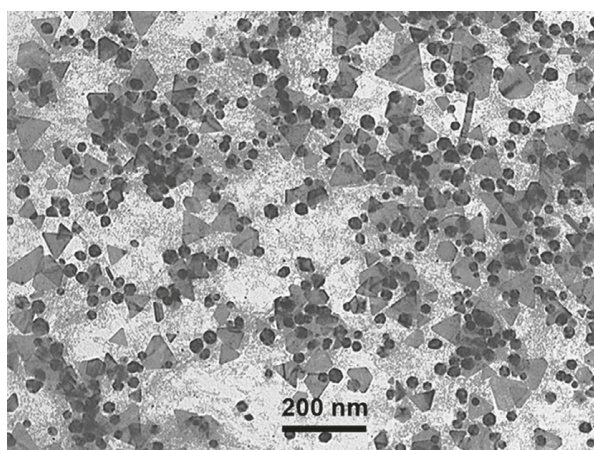

(a)

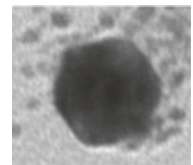

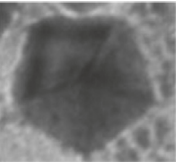

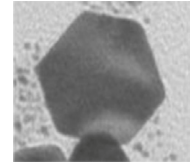

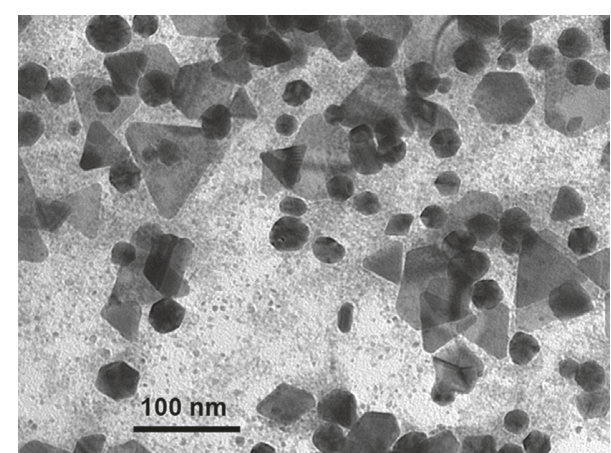

(b)
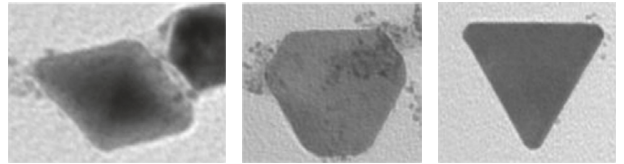

(c)

FIgURE 1: (a) TEM image of a typical distribution of AuNPs (molar ratio $R=2.74$ ) as produced with our synthesis route; (b) magnified image of a region of (a) where the small spheroidal nanoparticles are evidenced; (c) the most frequent shapes of the bigger nanoparticles.

depth: $10 \mathrm{~mm}$ ). The OD per volume unit was then $12 \mathrm{~mL}^{-1}$. The sample was irradiated with a collimated beam whose spot diameter was $5 \mathrm{~mm}$, using 4 different light intensities, namely, $2.6,1.32,0.6$, and $0.3 \mathrm{~W} \cdot \mathrm{cm}^{-2}$. Between any heating tests, the cavity was cooled down with pure $\mathrm{mQ}$ water at ambient temperature.

\section{Results and Discussion}

A typical TEM image of the produced nanoparticles is shown in Figure 1(a). They are widely polydispersed in shape and sizes. All these varieties are associated with the massive presence of small $(\sim 5 \mathrm{~nm}$ diameter $)$ spheroidal particles (Figure 1(b)). We have identified at least six typologies of bigger nanoparticles that are mostly present in the colloidal suspension (Figure 1(c)), while their dimensions range approximately from 30 to $80 \mathrm{~nm}$. The triangle-shaped AuNPs are the most frequently present, with an average edge dimension of $48 \pm 18 \mathrm{~nm}$.

In spite of this seemingly complex scenario, the spectra (Figure 2) for different values of $R$ are rather simple and exhibit the following main features:

(a) There are two absorption peaks $M_{\text {Green }}$ and $M_{\text {NIR }}$ centered in the green and NIR regions of the spectra.

(b) $R$ changes do not affect $M_{\text {Green }}$ position significantly. On the contrary, from $R=1.72$ to 2.15 , there is a moderate redshift of the maximum position of the NIR peak $M_{\text {NIR }}$, corresponding to a wavelength change from $805 \mathrm{~nm}$ to $825 \mathrm{~nm}$. Further increases of $R$, which are supposed to promote a more intense shift towards greater wavelengths $(\sim 1000 \mathrm{~nm})$, as suggested in [11], are found on the contrary to determine a slight blueshift of the spectrum.

(c) An interesting characteristic is the increase of the absorbance maxima $M_{\text {Green }}$ and $M_{\mathrm{NIR}}$, shifting from $R=1.7$ to $R=2.75$, namely, 1.2 -fold for $M_{\text {Green }}$ and

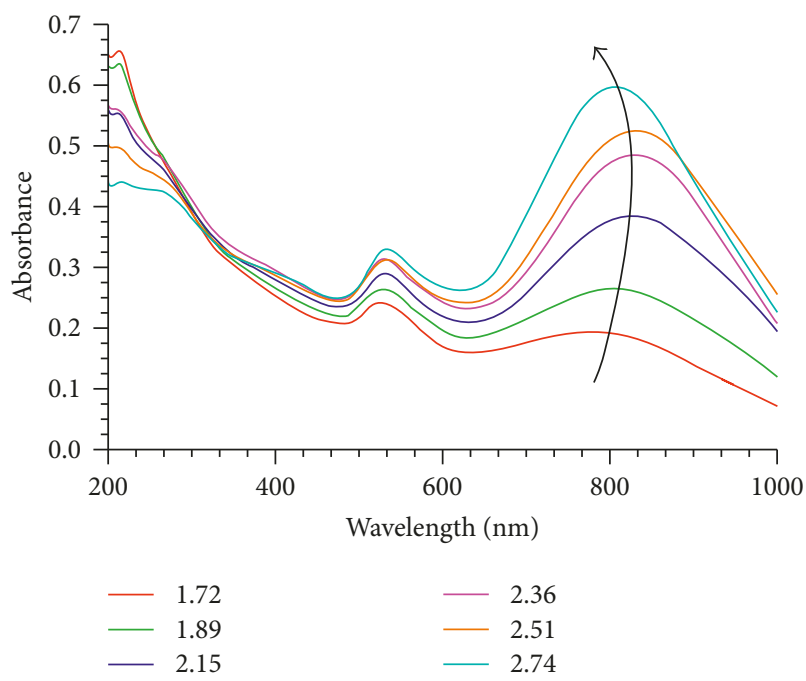

FIgURE 2: Evolution of the absorbance for several molar ratios $R$ after the synthesis. The curved arrow denotes the stop-and-return tendency of the NIR maxima.

3-fold for $M_{\mathrm{NIR}}$. This shows that, at least within a limited range of $R$ values, higher Au concentrations correspond to a higher chance that the reaction pathway will be directed towards the formation of a larger number of nanoparticles with plasmonic absorption in the NIR region rather than in the green region.

When dealing with the production of nanoparticles, tailoring their shape to match a designed ensemble behavior, for instance, optical absorption, is a demanding issue. Unfortunately, contrarily to other refined preparations in which the spectral features can be theoretically predicted, in our case, given the high polydispersivity of the colloidal suspensions, this is not possible. Nevertheless, considering 


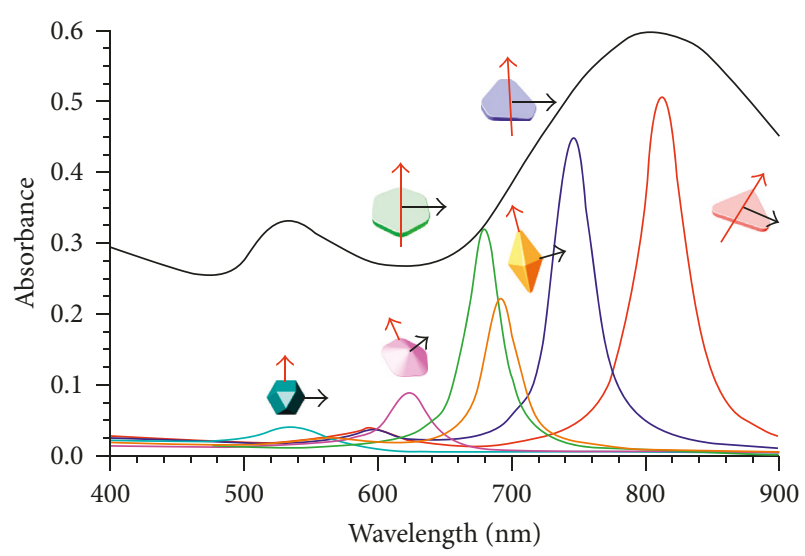

FIGURE 3: Simulation of the electromagnetic absorbance spectra of different species of nanoparticles that compose the experimental spectrum obtained with molar ratio $R=2.74$. Black arrow: direction of the impinging radiation; red arrow: electric field vector.

the typical shapes and dimensions of the AuNPs produced in this way, some peculiar qualitative information can be theoretically inferred by exploiting the results of the TEM analysis, to build up an electromagnetic model and calculate the absorbance spectrum as the result of the envelope of the spectra of the different typologies of nanoparticles. The interaction of the nanoparticles with the electromagnetic field was modeled by using COMSOL Multiphysics ${ }^{\circledR}$ software considering water as the host medium, while data from Johnson and Christy [18] have been used to model the dielectric constant of gold. A plane wave was considered as the illuminating electromagnetic field.

The results of the simulation are shown in Figure 3, where experimental absorbance of a sample obtained with $R=2.74$ is shown together with the calculated spectra of the aforementioned typologies of nanoparticles composing the colloidal suspension. The calculation highlights that nanoparticles with different shapes contribute to the overall absorbance with narrow bands peaked at different wavelengths, thus showing that the broadening of the spectrum is largely attributable to their envelope as a result of the high polydispersivity of the colloid. In the reported case, the experimental spectrum exhibits the NIR maximum absorbance around $820 \mathrm{~nm}$. The simulation also shows that the occurrence of such maximum can be attributed to the dipolar resonances of the $\mathrm{Au}$ triangular nanoplates indeed observed in the TEM images, in agreement with previous results [19-21]. Modeling also evidences that the resonance peak position is strictly related to the aspect ratio (AR) of the nanoparticle, defined as the ratio between its maximum and the minimum dimensions. As a general rule, higher $\mathrm{AR}$ corresponds to resonances at higher wavelengths, while approaching to minimum $\mathrm{AR}$, namely, 1 , corresponds to blueshift of the spectra around $530 \mathrm{~nm}$, the well-known dipolar resonance of spherical nanoparticles, which are nicely reproduced for the truncated cuboctahedron particle. Another outstanding output of the electromagnetic modeling is that the absorbance for each family of AuNPs is almost equal to the absorption. This result is related to the

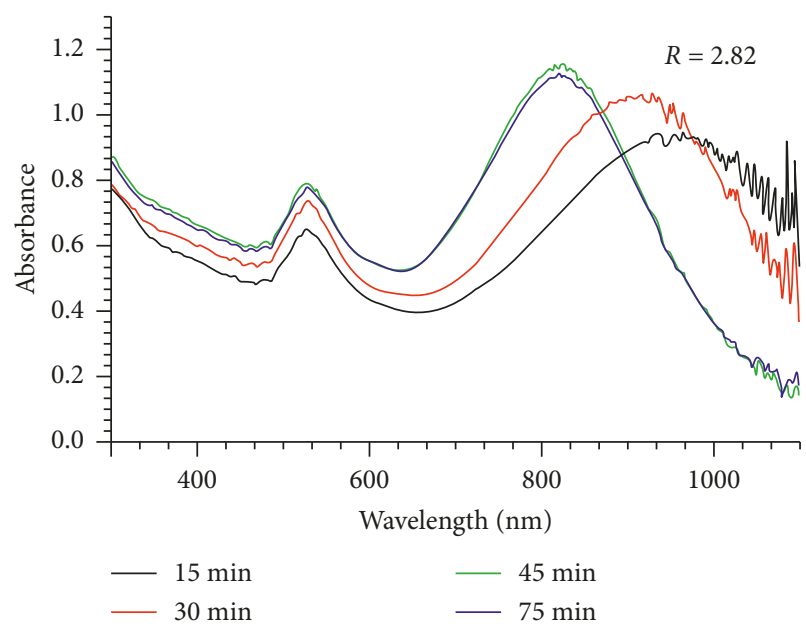

Figure 4: Evolution of the absorbance spectra of the colloidal suspension with $R=2.82$ at different times, reported in the figure, after the addition of the reducing $\mathrm{Na}_{2} \mathrm{~S}_{2} \mathrm{O}_{3}$ solution.

dimensions of the AuNPs obtained here in comparison with resonance wavelength, to such an extent that the contribution of the scattering turns out to be negligibly small. The obvious consequence is that light power absorbed at a given wavelength is only related to the mass amount of AuNPs that are resonant at that wavelength.

A first set of tests was performed to establish the reaction time at room temperature $\left(25^{\circ} \mathrm{C}\right)$ and to check the stability of the product, an expected intrinsic feature of the raw colloid. Unlike the most popular synthesis protocols, where proper surfactants are usually needed to generate and stabilize nanoparticles, the reduction based on the use of sodium thiosulfate exploits the same chemical species that induce nonspherical growth and intrinsically favor the stabilization of AuNpPs. Indeed, the $\mathrm{Na}_{2} \mathrm{~S}_{2} \mathrm{O}_{3}$ solution is a complex system, containing many sulfur-based compounds other than $\mathrm{SO}_{4}{ }^{2-}$ and $\mathrm{SO}_{3}{ }^{2}$ that are just present in the main reaction chain. These compounds, such as $S^{2-}$ and $S^{0}$, introduced by the self-modification of sodium thiosulfate, may convert into other sulfur derivatives [22], some of them working as capping reagents or stabilizers, which trigger anisotropic crystal growth and are also expected to stabilize AuNPs. However, given the complexity of the reaction frame that can be very sensitive to local production environment, we think that any assessment regarding this critical issue requires a dedicated prolonged observation of the colloidal suspension.

A typical example for time evolution of the reaction, referred to $R=2.82$, is reported in Figure 4. The two maxima $M_{\text {Green }}$ and $M_{\text {NIR }}$ show a different behavior as a function of time. The absorption maximum around $530 \mathrm{~nm}$ does not shift over time. On the contrary, after $15 \mathrm{~min}$ from the addition of the reducing $\mathrm{Na}_{2} \mathrm{~S}_{2} \mathrm{O}_{3}$ solution, the NIR band, located around $950 \mathrm{~nm}$, undergoes a blueshift for the following $45 \mathrm{~min}$, reaching a stable position at around $810 \mathrm{~nm}$ after $75 \mathrm{~min}$. This clearly shows reshaping of the nanoparticles that may lead to the variation in both the particles' $\mathrm{AR}$ and their geometrical shape. For instance, in the case of 


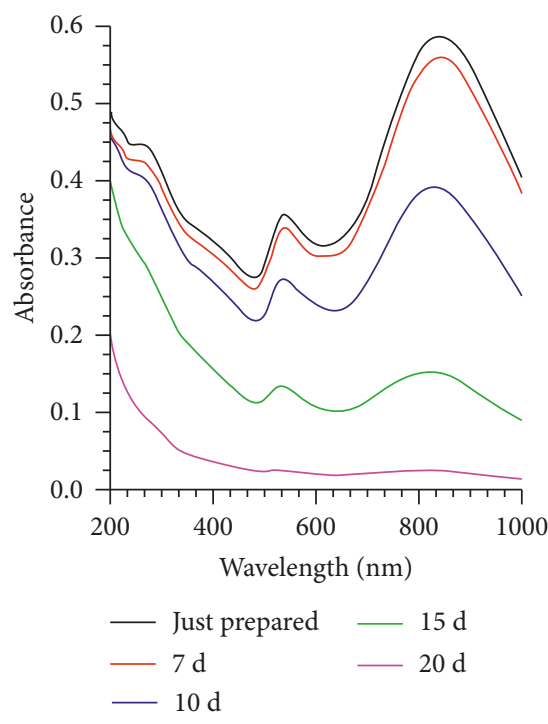

(a)

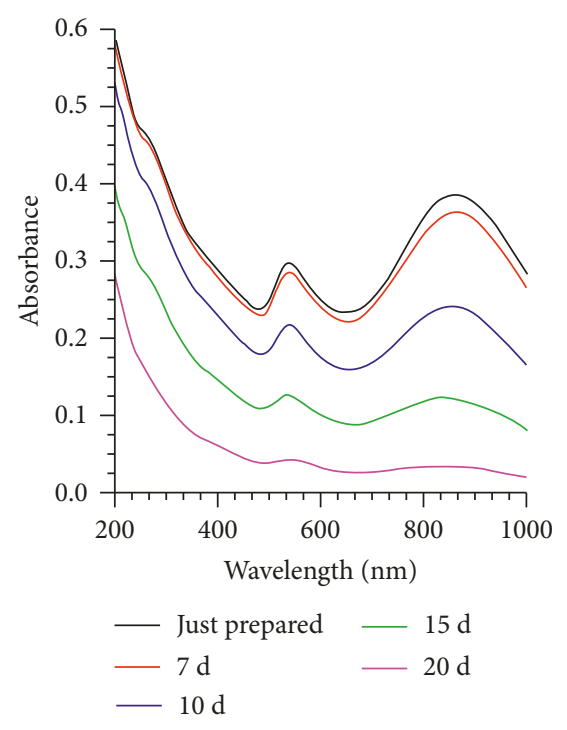

(b)

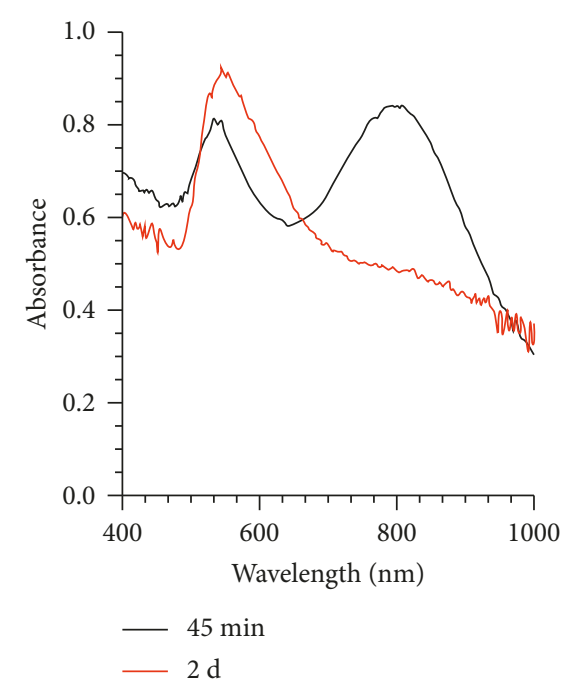

(c)

Figure 5: Time stability of naked AuNPs obtained for different molar ratios: (a) $R=2.74$, (b) $R=2.16$, and (c) $R=3.5$.

prismatic nanoparticles, they evolve from prisms with three concurrent edges to tip-truncated nanoprisms or nanodisks [19] with a corresponding variation in the wavelength of plasmonic resonance.

By prolonging the observation time, we have seen that the colloidal nanoparticles with $R<2.7$ maintain $M_{\mathrm{NIR}}$ typically within $\pm 5 \%$ for storage times not higher than 1 week (Figure 5). After that time, the colloid starts to flocculate and collapses, with no chance to redisperse the nanoparticles even with prolonged sonication. For $R>3$, this happens much more quickly, and AuNPs show a marked tendency to reshaping into round nanoparticles after only 48 hours, as shown in Figure 5 for $R=3.5$. Even if the reasons of this behavior are yet to be clarified, we guess that the effect can be attributed to Ostwald ripening, due to the intrinsic thermodynamic instability of the colloid at high $R$. In the first step, spheroidal nanoparticles decorate the platelets, switching to a successive process of aggregation in a shape that minimizes free energy [11]. This valuable intrinsic stability of naked NPs allows us to safely wait till the end of the reaction before proceeding to chitosan capping. This is an important outcome with respect to more conventional preparations pointed at bio-oriented applications.

In that case, surfactants used to promote asymmetric growth are usually toxic substances, like the widely used $\mathrm{CTAB}$, that must be mandatorily removed. Uncapped nanoparticles become very unstable and must be protected with nontoxic moieties in the shortest times, an operation that typically suffers problems of colloid precipitation just during the stabilization. $M_{\mathrm{NIR}}$ wavelengths of chitosancapped NPs (CC-AuNPs) follow the same stop-andreturn trend of naked NPs as shown in Figure 6(a), where spectra for $R$ up to 2.74 are reported. Instead, spectra related to further increase of $R$ (Figure 6(b)) show a decrease of NIR maximum absorbance and, for $R>3.1$, an abrupt pronounced NIR band broadening occurs. At the same time, as $R$ increases, two absorption bands in the UV region become more pronounced. These bands, peaking at 220 and $312 \mathrm{~nm}$, are likely to originate from absorption of the nonreacted $\mathrm{HAuCl}_{4}(217 \mathrm{~nm})$ and $\mathrm{Na}_{2} \mathrm{~S}_{2} \mathrm{O}_{3}(278 \mathrm{~nm})$ [23]. The results reported in [11], by following a similar synthesis, show a significant NIR shift up to $1100 \mathrm{~nm}$ for $R=2.4$, smaller than that one, $R=3.2$, needed to obtain an analogous behavior. We attribute this different evolution of the reaction to its inherent complexity and to the consequent sensitivity to local environmental conditions.

A comparison of the spectra of CC-AuNPs with naked NPs is shown in Figure 7 for $R=1.72,2.15$, and 2.74. At the first glance, a systematic redshift of the maxima of approximately $\sim 35 \mathrm{~nm}$ for the NIR band and $10 \mathrm{~nm}$ for the green band is observed. This is a first clue that the colloidal NPs have been correctly capped [24].

However, the most significant differences between naked and CC-AuNPs can be more precisely appreciated only after a proper decomposition of the experimental spectra into separate spectral components. These subbands are chosen to be Lorentzian-shaped, characterized by an absolute maximum and a full width at half maximum (FWHM). A typical example that shows the accuracy of the reconstruction of the experimental spectra with the various bands is shown in Figure 8, in the case of naked and CC-AuNPs, respectively, synthesized with $R=2.74$. The residuals between the experimental trace and the reconstructed spectrum do not exceed 0.005 units of absolute absorbance in all the measured spectra.

Four of the five subbands that appear in the spectra of the naked and capped version of AuNPs as well can be ascribed to (i) the presence of unreacted $\mathrm{HAuCl}_{4}$ and $\mathrm{Na}_{2} \mathrm{~S}_{2} \mathrm{O}_{3}$ (resp., bands A and B); (ii) spheroidal nanoparticles (D); (iii) asymmetrical nanoparticles (E). The band C developed at $400 \mathrm{~nm}$ can be attributed to the coalescence of the two interband transitions of gold at 330 and $470 \mathrm{~nm}$, which are 


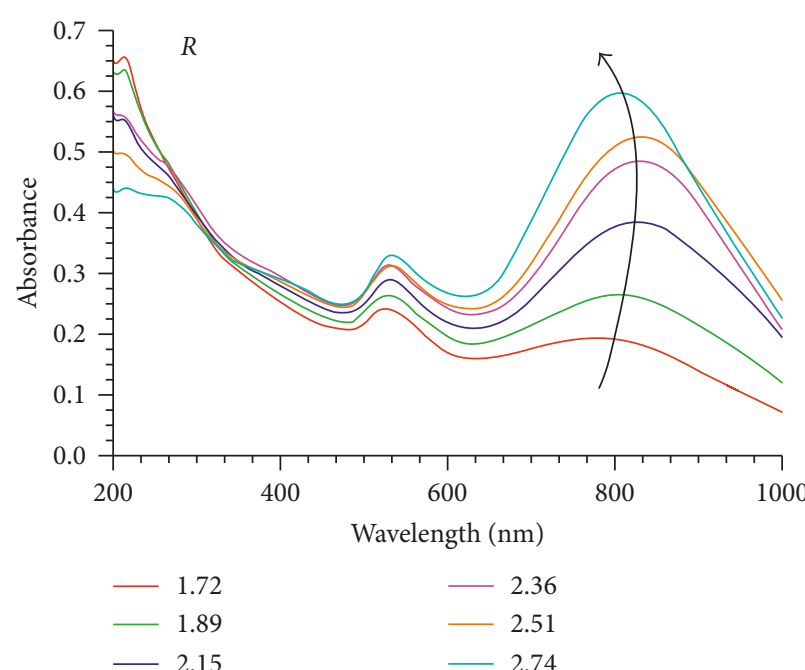

(a)

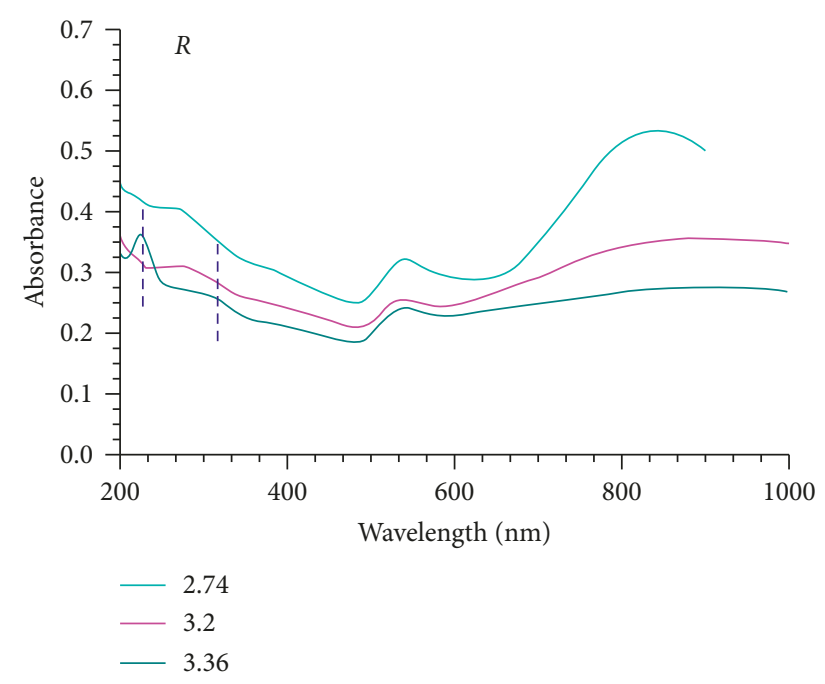

(b)

Figure 6: Evolution of the absorbance spectra of chitosan-capped NPs (CC-AuNPs) synthesized at different $R$ values. (a) The stop-andreturn behavior found for $R$ ranging from 1.72 to 2.74. (b) Absorption bands for R ranging from 2.74 to 3.36 .

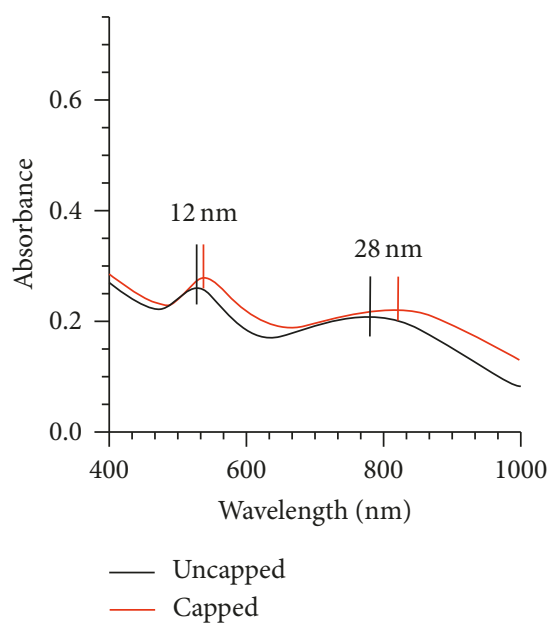

(a)

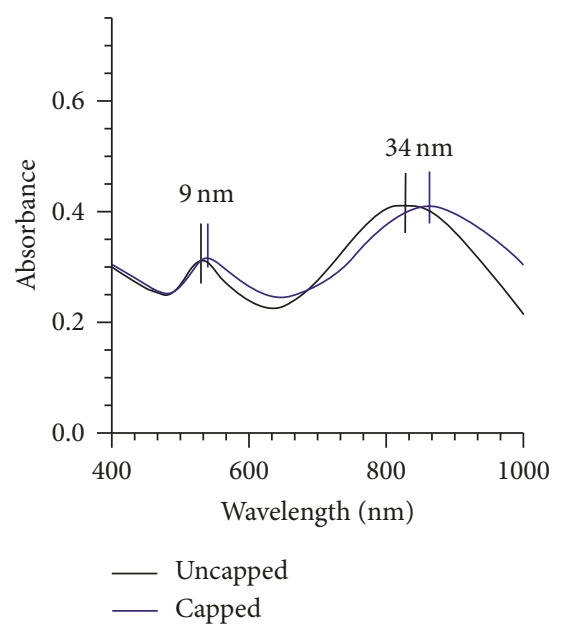

(b)

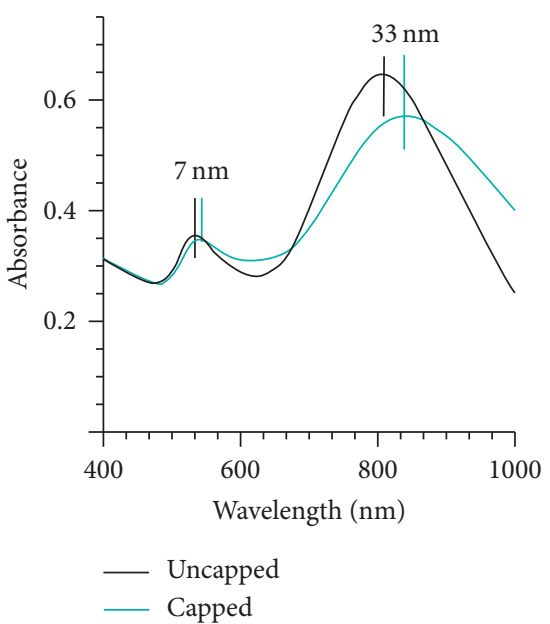

(c)

Figure 7: Spectra of samples of AuNPs before and after the addition of chitosan solution for three molar ratios: (a) $R=1.72$, (b) $R=2.15$, and (c) $R=2.74$.

just barely resolved in the imaginary part of its dielectric constant [25] and appear as a unique band in our decomposition.

The redshift of the green and NIR bands due to the capping process is evident in Figure 9(a), where peak wavelength versus $R$ is reported. Its value, averaged on the whole $R$ range for the NIR band of the decomposed spectra, is $35 \pm 5 \mathrm{~nm}(14 \%)$, while a smaller shift $6.3 \pm 0.8 \mathrm{~nm}(13 \%)$ is obtained for the green band. Low fluctuations in the wavelengths corresponding to the deconvoluted maxima for NIR and green bands $M_{\mathrm{NIR}}{ }^{\text {dec }}$ are $M_{\mathrm{Green}}{ }^{\mathrm{dec}}$, respectively, are a clear sign of the capping process solidity, meaning that high variations in the added $\mathrm{Na}_{2} \mathrm{~S}_{2} \mathrm{O}_{3}$ corresponding to the $R$ ranging from 1.72 to 2.74 , do not lead to significant variations in the wavelength shift for both nanospheres and anisotropic nanoparticles under the same reaction conditions.

As anticipated, the redshift is the first indication of a correct capping, but another concurrent mechanism can induce a spectral shift in the same direction, namely, the interparticle electromagnetic coupling [10].

Indeed, its presence can be inferred by the further broadening of the spectra reported in Figure 9(b). The average value of FWHM switches from an average of $320 \mathrm{~nm}$ for the uncapped NPs to $420 \mathrm{~nm}$ for the chitosan-capped NPs. The occurrence of this mechanism can be attributed to the binding process of chitosan to the as-synthesized AuNPs. A chitosan unit is a long-chain polymer exposing $\mathrm{NH}_{2}$ groups that establish affine binding to gold [26]. As amine 


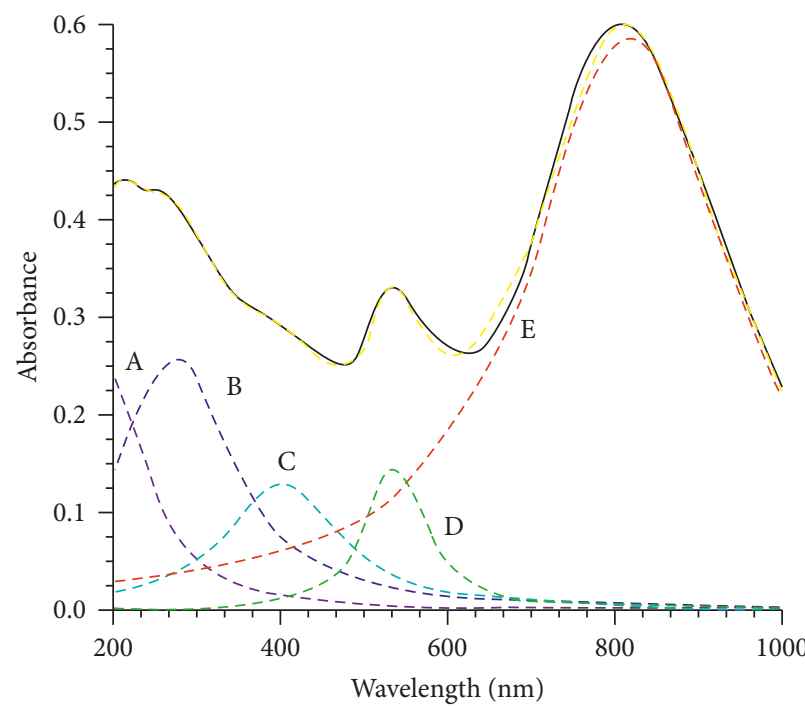

(a)

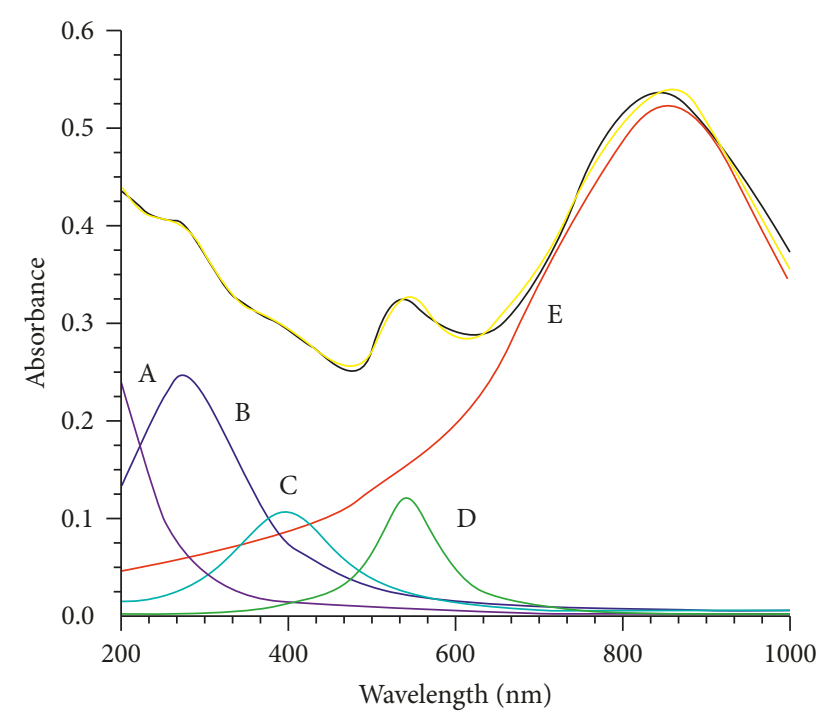

(b)

Figure 8: Experimental and decomposed absorbance spectra in the case $\mathrm{R}=2.74$ for uncapped (a) and capped (b) AuNPs. Black line: experimental spectrum. Yellow line: spectrum reconstructed by adding up the decomposed spectral bands A, B, C, D, and E.

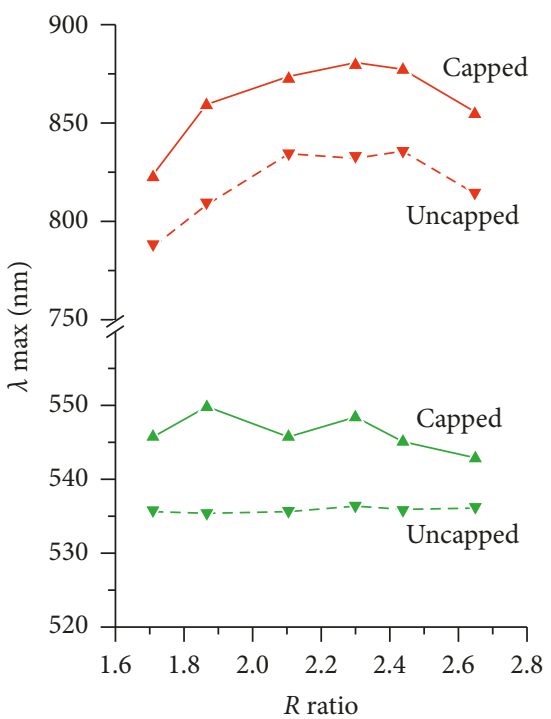

(a)

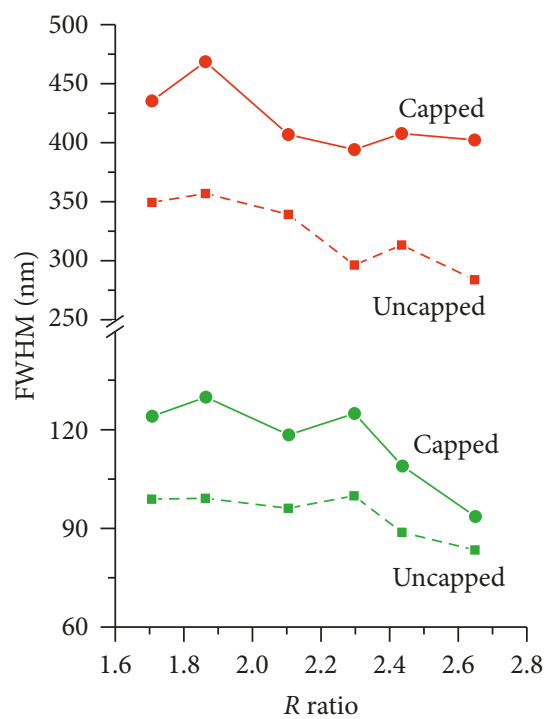

(b)

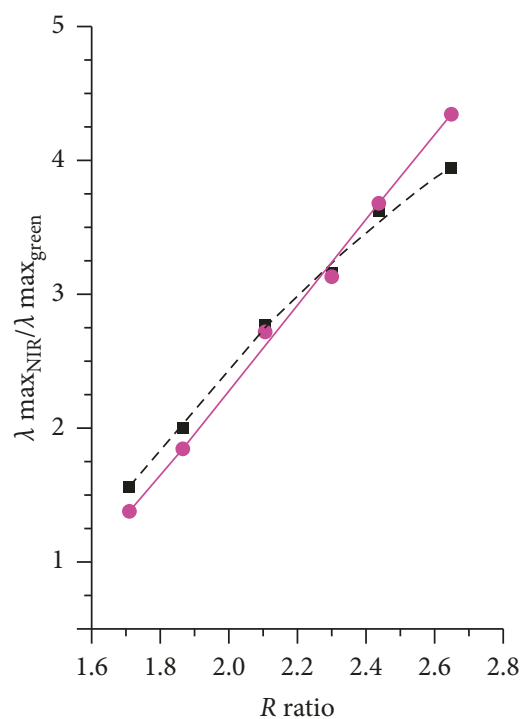

- Uncapped

- Capped

FIGURE 9: Results of the decomposition of the experimental spectra versus the molar ratio $R$ before and after the chitosan capping. (a) Wavelength of peak absorbances; (b) FWHM of the NIR- and green-tuned bands; (c) ratio between the maxima in the NIR band $\left(M_{\mathrm{NIR}}\right)$ and in the green band $\left(M_{\mathrm{Green}}\right)$. The straight lines are only a guide for the eye.

groups are distributed all along the molecular chain, a chitosan unit can graft to a single NP generating a protective shell (clearly visible in Figure 3 of [14]), but in principle, it can also bind to several nanoparticles that can thus move in close proximity, with consequent switching of the electromagnetic coupling mechanism.

Imbalance between the population of NIR-resonating and green-resonating nano-objects was estimated by calculating the ratio $M_{\mathrm{NIR}}{ }^{\mathrm{dec}} / M_{\mathrm{Green}}{ }^{\mathrm{dec}}$ between the maxima of the decomposed bands versus $R$ (Figure 9(c)). The results show that there is a linear increasing trend with minor differences between naked and CC-AuNPs. In the latter case, which also constitutes the practical goal, there is approximately a 3-fold increase of $M_{\mathrm{NIR}}{ }^{\mathrm{dec}} / M_{\mathrm{Green}}{ }^{\mathrm{dec}}$ switching from $R=1.75$ to $R=2.74$, while, at this latter value of $R$, the ratio increases from 4 for the naked particles to 4.34 for the CC-AuNPs. Taking into account the reproducibility of the synthesis process, this can be considered a physiological fluctuation as discussed later on. 


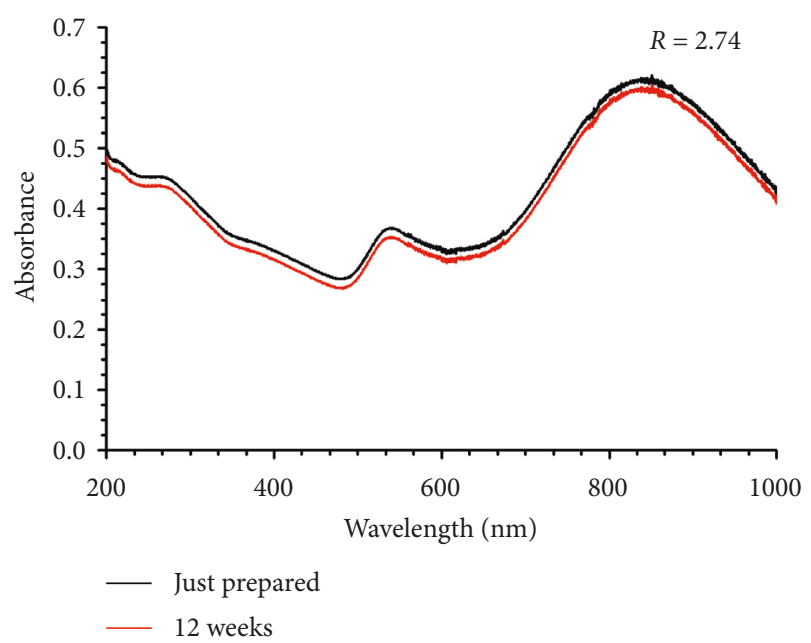

(a)

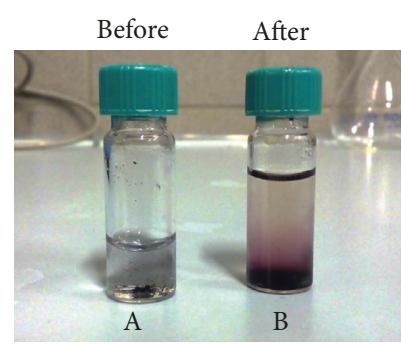

(b)

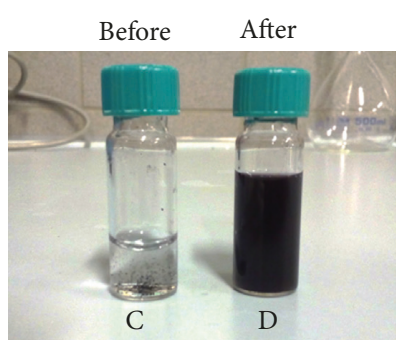

(c)

FIgURe 10: (a) Spectra of colloidal solutions of CC-AuNPs synthesized with $R=2.74$. Pictures of 12 -week samples of naked AuNPs (b) and CC-AuNPs (c) before (A, C) and after (B, D) 5 min sonication.

As further verification of the successful capping, we measured the $\zeta$-potential of colloidal suspensions. They exhibit a typical shift from $-35 \mathrm{mV}$ to $+40 \mathrm{mV}$, with a tolerance $\pm 10 \%$ on both values, confirming the correct coverage of colloidal nanoparticles, in agreement with previous results [14].

Capped nanoparticles have a much higher colloidal stability than that one of the as-made colloid. Sonication of suspensions after 12 weeks of aging produced a rapid redispersion of the colloid that exhibits absorbance spectra similar to the ones recorded immediately after capping (Figure 10).

Indeed, only a small redshift of the NIR band $(\sim 3 \mathrm{~nm})$ was observed along with a slight decrease of absorbance $(\sim 0.02)$. The different stability of aged colloids is also well highlighted in the photos of 12-week colloidal samples of naked and CC-AuNPs shown in the same figure before and after the sonication process. One can see the complete redispersion of the colloid in the latter case and a substantial insensitivity to the mechanical stimulus in the latter case. Even if we were not able to extend the observation as in [7], the slight variation in the absorbance maximum seems to indicate a favorable behavior on longer aging times as well.

The reproducibility of the final products in our framework was tested by repeating the synthesis and capping protocol 5 times in the case of $R=2.74$. Maximum absorbance in the NIR band has a typical oscillation of \pm 0.01 , while the wavelength of absorption maximum oscillates within $\pm 7 \mathrm{~nm}$ around $810 \mathrm{~nm}$ (data not shown).
Finally, we tested CC-AuNPs as optothermal transducers. With this goal in mind, we considered CC-AuNPs obtained with $R=2.74$, as at this value, we observed the highest value of $M_{\mathrm{NIR}}{ }^{\mathrm{dec}} / M_{\mathrm{Green}}{ }^{\mathrm{dec}}$ ratio, and thus, it is expected to give the highest performances. The results of the experimental tests are embedded in Figure 11. As shown in the lower part of the figure, even at moderate light intensities, hyperthermic regimes $\left(T>50^{\circ} \mathrm{C}\right)$ are quickly reached: for instance, at $1.3 \mathrm{~W} \cdot \mathrm{cm}^{-2}$, this temperature is obtained only after $26 \mathrm{~s}$.

The heating speed of the colloid (a very important issue when dealing with a clinically oriented application) is calculated by best fitting the thermal kinetics of temperature versus time and taking the time derivative evaluated at $t=0$, namely, $r$, that proportionally depends on power density $I$. The result, reported in the upper inset of Figure 11, shows that the ratio $r / I$ versus $I$ is almost constant and equal to $1.04^{\circ} \mathrm{C} \mathrm{cm}{ }^{2} \cdot \mathrm{W}^{-1} \cdot \mathrm{s}^{-1}$. Thus, by themselves, these data assure that our colloidal system is an excellent optothermal transducer. Nevertheless, the comparison with the optimized performances expected after a final purification as performed by Zhang et al. [11-14] is mandatory, as their results represent the most meaningful benchmark for our work.

However, as the experimental conditions given in that case (optical density and input beam intensity) differ from ours, a proper common figure of merit must be identified to correctly compare the respective performances. In order to reach this goal, we chose to consider the molar heating rate (MHR), which has been recently introduced to evaluate the 


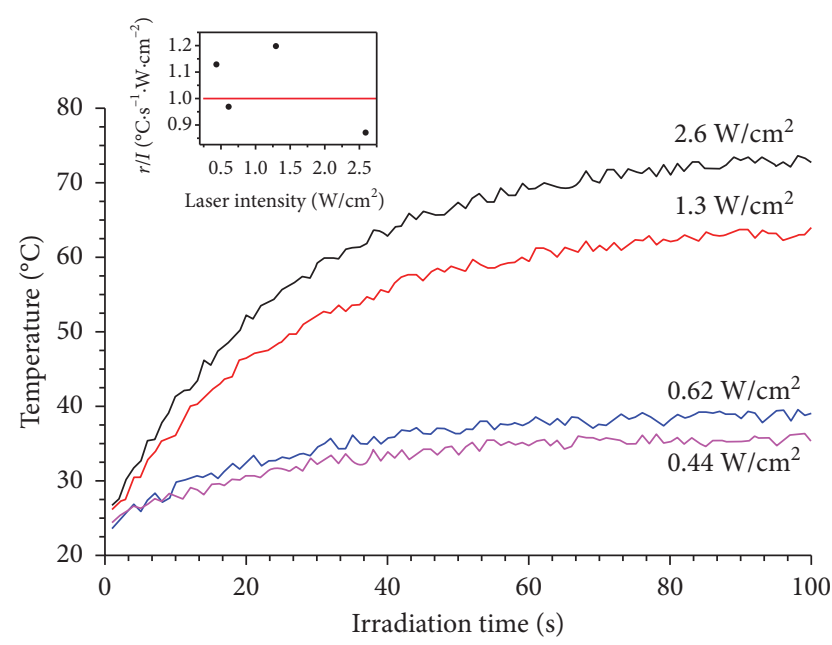

FIGURE 11: Thermal kinetics at different light intensities obtained irradiating a colloidal sample fabricated with $R=2.74$ with an OD of $12 \mathrm{~mL}^{-1}$ at different light intensities (I). Upper inset: initial temperature increases $r$ normalized with the input light intensity $I$.

photothermal properties of different nanostructures in the biomedical realm [27]. This quantity was defined as the initial rate of change $r$ divided by the Au molar concentration, which is missing among the data presented in [14]. Hence, we exploited the known optical density $\left(\mathrm{OD}\left(\mathrm{mL}^{-1}\right)\right)$, which is proportional to the concentration. The final step was the normalization of MHR to the input beam intensity $I$, because, as experimentally evidenced (Figure 11), the rate $r$ scales proportionally with it.

Thus, the comparison between MHRs may be suitably done by evaluating the ratio:

$$
\frac{\mathrm{MHR}_{1}}{\mathrm{MHR}_{2}}=\frac{r_{1} \cdot \mathrm{OD}_{2} \cdot I_{2}}{r_{2} \cdot \mathrm{OD}_{1} \cdot I_{1}}
$$

where the indices 1 and 2 refer to our colloid and the one of [14], respectively. Considering the case with $I_{1}=2.6 \mathrm{~W} \cdot \mathrm{cm}^{-2}$ and $\mathrm{OD}_{1}=12 \mathrm{~mL}^{-1}$, the rate $r_{1}$ results to be $2.1^{\circ} \mathrm{C} \mathrm{s}^{-1}$. Inserting (1) the above values and the other involved parameters $\left(I_{2}=1 \mathrm{~W} \cdot \mathrm{cm}^{-2}, r_{2}=0.17^{\circ} \mathrm{C} \mathrm{s}^{-1}\right.$, and $\left.\mathrm{OD}_{2}=1 \mathrm{~mL}^{-1}\right)$, an improving factor of $\sim 2.5$ is obtained for the purified colloid. This enhancement can be partially explained by the heat transfer properties of the colloid. In fact, a given amount of Au mass enriched by nanotriangles almost completely transforms the radiation in heat, while, in the nonenriched colloid, an equal mass of nanotriangles is unavoidably associated with the presence of a passive mass of spheroidal nanoparticles that simply increases the thermal capacity of the colloid without absorbing radiation, decreasing the overall heat transfer between the AuNPs to the environment.

Similar enhancements are found considering the thermal kinetics recorded at other intensity levels, confirming the enhanced performance of the purified colloid. Although numerical evaluation confirms the expected better thermooptical performances of the purified colloid, the practical advantage of nanoparticles filtering is, however, debatable. Indeed, as already mentioned, this enhancement is obtained at expenses of extraprocessing centrifugation steps to purify and concentrate the NIR-tuned nanoparticles. On the other hand, it is well known that these processes are highly critical, as they suffer from issues of major loss of NIR-absorbing material and strong risks of coalescence, in spite of the presence of capped nanoparticles. Thus, the enriching procedure can likely become extremely cumbersome and time-consuming and, after all, considering the moderate enhancement achievable, not convenient for mass-oriented applications. On the contrary, our CC-AuNPs follow a much simpler preparation route, yet maintaining excellent photothermal properties.

In recent years, a number of different kinds of NIRabsorbing nanoparticles have been synthesized for photothermal cancer therapy. A comparison with such colloidal systems can be useful to further point out the advantages of our colloid. We have considered nanostars (AuNSs) and nanorods (AuNRs), whose optothermal performances have been in-depth detailed in [27], and that we have chosen them as further benchmark for our colloidal system. In all of the compared cases, our colloid shows better performances with respect to the nanoparticles therein reported. For instance, in the case of long-gold nanorods (L-GNRs), tuned on a working wavelength of $821 \mathrm{~nm}$, the MHR of our colloid is 5.2 times higher, while it is 10 times higher than the MHR of AuNSs resonating at $829 \mathrm{~nm}$. A dedicated analysis would be necessary to clarify the origin of these better performances, but, nevertheless, we speculate that a possible reason lies in the different thermal capacity of NIR-absorbing species. For instance, considering the population of nanotriangles obtained from our synthesis, whose average edge is $46 \mathrm{~nm}$, and assuming a thickness of $8.5 \mathrm{~nm}$ [11], its thermal capacity comes out to be 1.6 times lower that one reported for the L-GNRs described in [27]. Even if this does not prove as a conclusive explanation (that would require the solution of time-dependent heat equations with convective boundary conditions), it explains qualitatively the faster heat transfer between AuNPs and the surrounding medium and, ultimately, it accounts for a higher $r$. Finally, it is worth noticing that the comparison of our CC-AuNPs with AuNRs and AuNSs is still more favorable when considering their preparation for biomedical applications, as the earlier mentioned widespread use of $C T A B$ to promote the nonspherical growth of AuNPs raises nontrivial substitution problems as previously discussed.

\section{Conclusions}

We have reported on the synthesis and characterization of a NIR-absorbing colloid prepared by the reduction of $\mathrm{HAuCl}_{4}$ with $\mathrm{Na}_{2} \mathrm{~S}_{2} \mathrm{O}_{3}$, followed by the stabilization with chitosan, a highly biocompatible polymer. This kind of reaction does not require any surfactant to promote the growth of nonspherical nanoparticles. The overall preparation procedure, also taking into account the overnight stabilization of the colloid, requires approximately 14 hours. We did not adopt any more steps aimed at concentrating NIR-absorbing AuNPs, but we rather focused on the performances of the raw product, never evaluated before. This is 
a mixture of chitosan-capped nanoparticles (CC-AuNPs) of various shapes and dimensions, with two characteristic absorbance bands located in the green and in NIR region of the spectrum. We have identified the molar ratio $R$ between $\mathrm{HAuCl}_{4}$ and $\mathrm{Na}_{2} \mathrm{~S}_{2} \mathrm{O}_{3}$ that allows the preparation of a colloid with the highest absorbance, namely, $R \sim 2.8$. As further fingerprints of a successful preparation, the spectrum of CCAuNPs must exhibit a redshift of the NIR maximum wavelength of $35 \pm 5 \mathrm{~nm}$, with a typical FWHM that we found not to exceed $470 \mathrm{~nm}$, while the $\zeta$-potential must shift from a negative value of $-35 \mathrm{mV}$ to a positive potential of $+40 \mathrm{mV}$. While it is relatively simple to prepare colloids with an optimized NIR absorbance around $810 \mathrm{~nm}$, further shifts towards the second biological transparency window $(\sim 1000 \mathrm{~nm})$ by varying the molar ratio invariably led to broad bands in the NIR with scarcely defined resonances. We attributed this behavior to a high sensitivity of the reaction itself to the local working conditions at higher molar ratios, as this reaction develops in a very complex chemical frame, so that the effective reproducible tuning of the NIR band at higher wavelengths based on the sole adjustment of $R$ as suggested in [11] does not seem so easily predictable. Optothermal properties of the nonrefined colloid have been tested, and a comparison with those ones of the refined product has been made by considering their molar heating rate (MHR). As expected, the refined version indeed yields better performances, but only with a moderately (2.5-fold) higher value than that one found for our nonenriched product. Moreover, considering other popular NIRresonating nanoparticles, namely, Au nanostars and nanorods, the MHR of our unrefined colloid results at least 5-fold higher, with further advantages represented by a much simpler synthetic route that does not require mandatory substitution of toxic surfactants that are usually used to promote the anisotropic growth. In conclusion, the presented colloidal system, even though unrefined, was shown to prove as a good trade-off between the straightforwardness of fabrication and optothermal performances, thus turning out as a competitive alternative for biomedical applications.

\section{Conflicts of Interest}

The authors declare that they have no conflicts of interest regarding the publication of this paper.

\section{Acknowledgments}

The authors wish to thank Dr. Roberto Olmi (Institute of Applied Physics "Nello Carrara" of the National Council of Researches) for his valuable help in the FLIR-assisted measurements.

\section{References}

[1] C. M. Cobley, L. Au, J. Chen, and Y. Xia, "Targeting gold nanocages to cancer cells for photothermal destruction and drug delivery," Expert Opinion on Drug Delivery, vol. 7, no. 5, pp. 577-587, 2010.
[2] T. Sun, Y. S. Zhang, B. Pang, D. C. Hyun, M. Yang, and Y. Xia, "Engineered nanoparticles for drug delivery in cancer therapy," Angewandte Chemie International Edition in English, vol. 53, no. 46, pp. 12320-12364, 2014.

[3] I. C. Sun, J. H. Na, S. Y. Jeong et al., "Biocompatible glycol chitosan-coated gold nanoparticles for tumor-targeting CT imaging," Pharmaceutical Research, vol. 31, no. 6, pp. 14181425, 2014.

[4] D. B. Chithrani, M. Dunne, J. Stewart, C. Allen, and D. A. Jaffray, "Cellular uptake and transport of gold nanoparticles incorporated in a liposomal carrier," Nanomedicine: Nanotechnology, Biology and Medicine, vol. 6, no. 1, pp. 161-169, 2010.

[5] M. R. Choi, K. J. Stanton-Maxey, J. K. Stanley et al., "A cellular Trojan Horse for delivery of therapeutic nanoparticles into tumors," Nano Letters, vol. 7, no. 12, pp. 3759-3765, 2007.

[6] S. J. Madsen, S. K. Baek, A. R. Makkouk, T. Krasieva, and H. Hirschberg, "Macrophages as cell-based delivery systems for nanoshells in photothermal therapy," Annals of Biomedical Engineering, vol. 40, no. 2, pp. 507-515, 2012.

[7] G. Margheri, A. Zoppi, R. Olmi et al., "Tumor-tropic endothelial colony forming cells (ECFCs) loaded with near-infrared sensitive Au nanoparticles: A "cellular stove" approach to the photoablation of melanoma," Oncotarget, vol. 7, no. 26, pp. 39847-39860, 2016.

[8] Y. Wang, K. C. L. Black, H. Luehmann et al., "Comparison study of gold nanohexapods, nanorods, and nanocages for photothermal cancer treatment," ACS Nano, vol. 7, no. 3, pp. 2068-2077, 2013.

[9] P. del Pino, G. de la Higuera, J. M. de la Fuente, and C. Tortiglione, "Gold nanoprisms for photothermal cell ablation in vivo," Nanomedicine, vol. 9, no. 13, pp. 1913-1922, 2014.

[10] J. F. Hainfeld, M. J. O’Connor, P. Lin, L. Qian, D. N. Slatkin, and H. M. Smilowitz, "Infrared-transparent gold nanoparticles converted by tumors to infrared absorbers cure tumors in mice by photothermal therapy," PLoS One, vol. 9, no. 2, Article ID e88414, 2014.

[11] G. Zhang, J. B. Jasinski, J. L. Howell, D. Patel, D. P. Stephens, and A. M. Gobin, "Tunability and stability of gold nanoparticles obtained from chloroauric acid and sodium thiosulfate reaction," Nanoscale Research Letters, vol. 7, no. 1, p. 337, 2012.

[12] C. Bao, N. Beziere, P. del Pino, B. Pelaz et al., "Gold nanoprisms as optoacoustic signal nanoamplifiers for in vivo bioimaging of gastrointestinal cancers," Small,vol. 9, no. 1, pp. 68-74, 2013.

[13] F. Tian, M. J. D. Clift, A. Casey et al., "Investigating the role of shape on the biological impact of gold nanoparticles in vitro," Nanomedicine, vol. 10, no. 17, pp. 2643-2657, 2015.

[14] G. Zhang, X. Sun, J. Jasinski, D. Patel, and A. M. Gobin, "Gold/chitosan nanocomposites with specific near infrared absorption for photothermal therapy applications," Journal of Nanomaterials, vol. 2012, Article ID 853416, 9 pages, 2012.

[15] M. J. Laudenslager, J. D. Schiffman, and C. L. Schauer, "Carboxymethyl chitosan as a matrix material for platinum, gold, and silver nanoparticles," Biomacromolecules, vol. 9, no. 10 , pp. 2682-2685, 2009.

[16] I. L. Wedmore, J. G. McManus, A. E. Pusateri, and J. B. Holcomb, "A special report on the chitosan-based hemostatic dressing: experience in current combat operation," Journal of Trauma: Injury, Infection, and Critical Care, vol. 60, no. 3, pp. 655-658, 2006.

[17] H. S. Zhou, I. Honma, H. Komiyama, and J. W. Haus, "Controlled synthesis and quantum-size effect in gold-coated 
nanoparticles," Physical Review B, vol. 50, no. 16, pp. 1205212056, 1994.

[18] P. B. Johnson and R. Christy, "Optical constants of the noble metals," Physical Review B, vol. 6, pp. 4370-4379, 1972.

[19] B. Pelaz, V. Grazu, A. Ibarra, C. Magen, P. del Pino, and J. M. de la Fuente, "Tailoring the synthesis and heating ability of gold nanoprisms for bioapplications," Langmuir, vol. 28, no. 24 , pp. 8965-8970, 2012.

[20] L. Soaresa, A. Csákid, J. Jatschkad et al., "Localized Surface Plasmon Resonance (LSPR) biosensing using gold nanotriangles: detection of DNA hybridization events at roomtemperature," Analyst, vol. 139, no. 19, pp. 4964-4973, 2014.

[21] Y. Ren, H. Qi, Q. Chen, S. Wang, and L. Ruan, "Localized surface plasmon resonance of nanotriangle dimers at different relative positions," Journal of Quantitative Spectroscopy \& Radiation Transfer, vol. 199, pp. 45-51, 2017.

[22] J. Y. Chen, S. Z. Yang, J. J. Ke, and M. H. Mao, Study and developments of Hydrometallurgy, Metallurgical Industry Press, Beijing, China, 1998.

[23] X. Liang, Z.-J. Wang, and C.-J. Liu, "Size-controlled synthesis of colloidal gold nanoparticles at room temperature under the influence of glow discharge," Nanoscale Research Letters, vol. 5, pp. 124-129, 2010.

[24] J. Liu and Y. Lu, "Colorimetric biosensors based on DNAzyme-assembled gold nanoparticles," Journal of Fluorescence, vol. 14, no. 4, pp. 343-354, 2004.

[25] P. G. Etchegoin, E. C. Le Ru, and M. Meyer, "An analytic model for the optical properties of gold," Journal of Chemical Physics, vol. 125, no. 16, article 164705, 2006.

[26] T. Sainsbury, T. Ikuno, D. Okawa, D. Pacile, J. M. J. Frechet, and A. Zettl, "Self-assembly of gold nanoparticles at the surface of amine- and thiol-functionalized boron nitride nanotubes," Journal of Physical Chemistry C, vol. 111, no. 35, pp. 12992-12999, 2007.

[27] W. Xiaocui, L. Guohua, D. Yu, and S. Shuqing, "Understanding the photothermal effect of gold nanostars and nanorods for biomedical applications," RSC Advances, vol. 4, no. 57, pp. 30375-30383, 2014. 

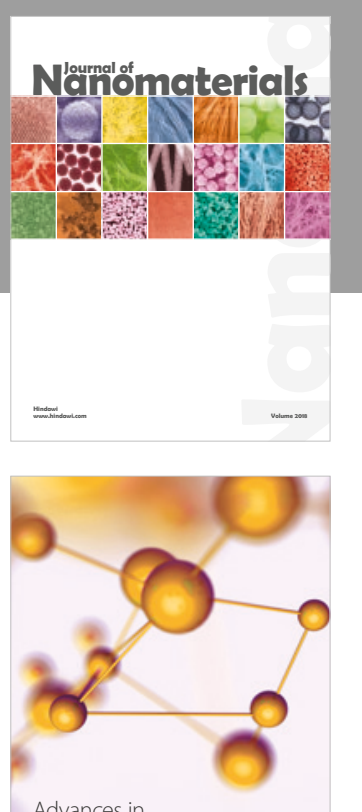

Physical Chemistry
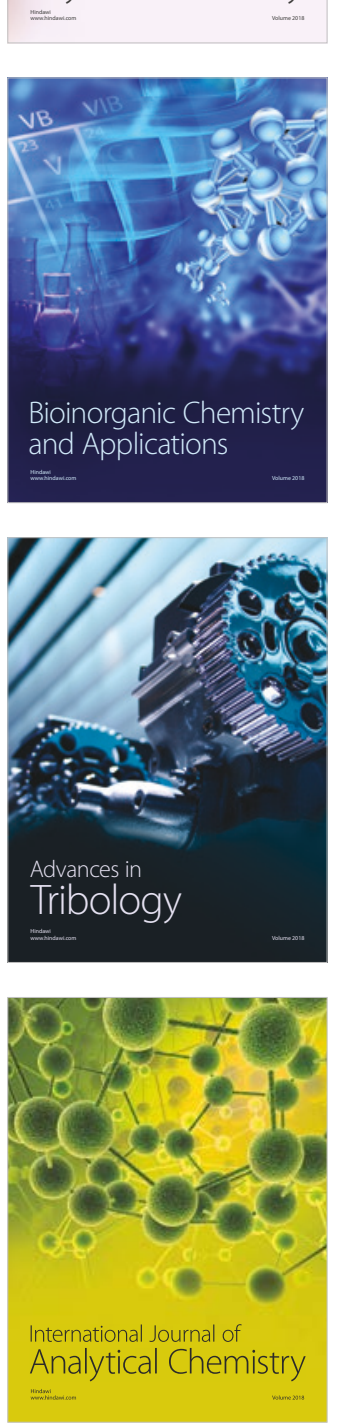

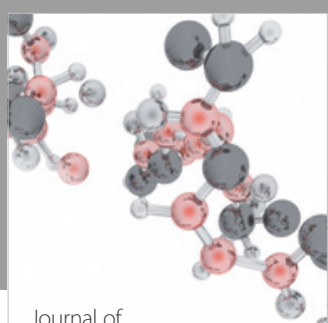

Analytical Methods

in Chemistry

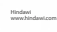

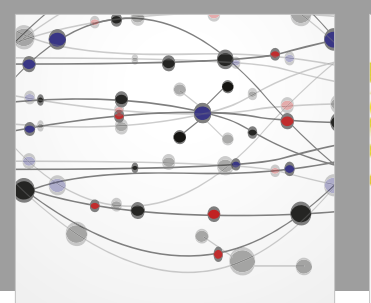

The Scientific World Journal

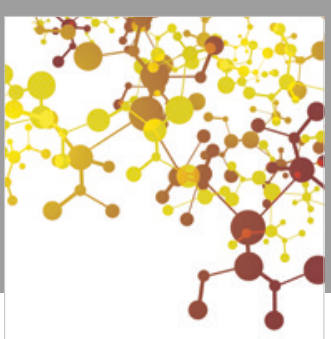

Journal of

Applied Chemistry
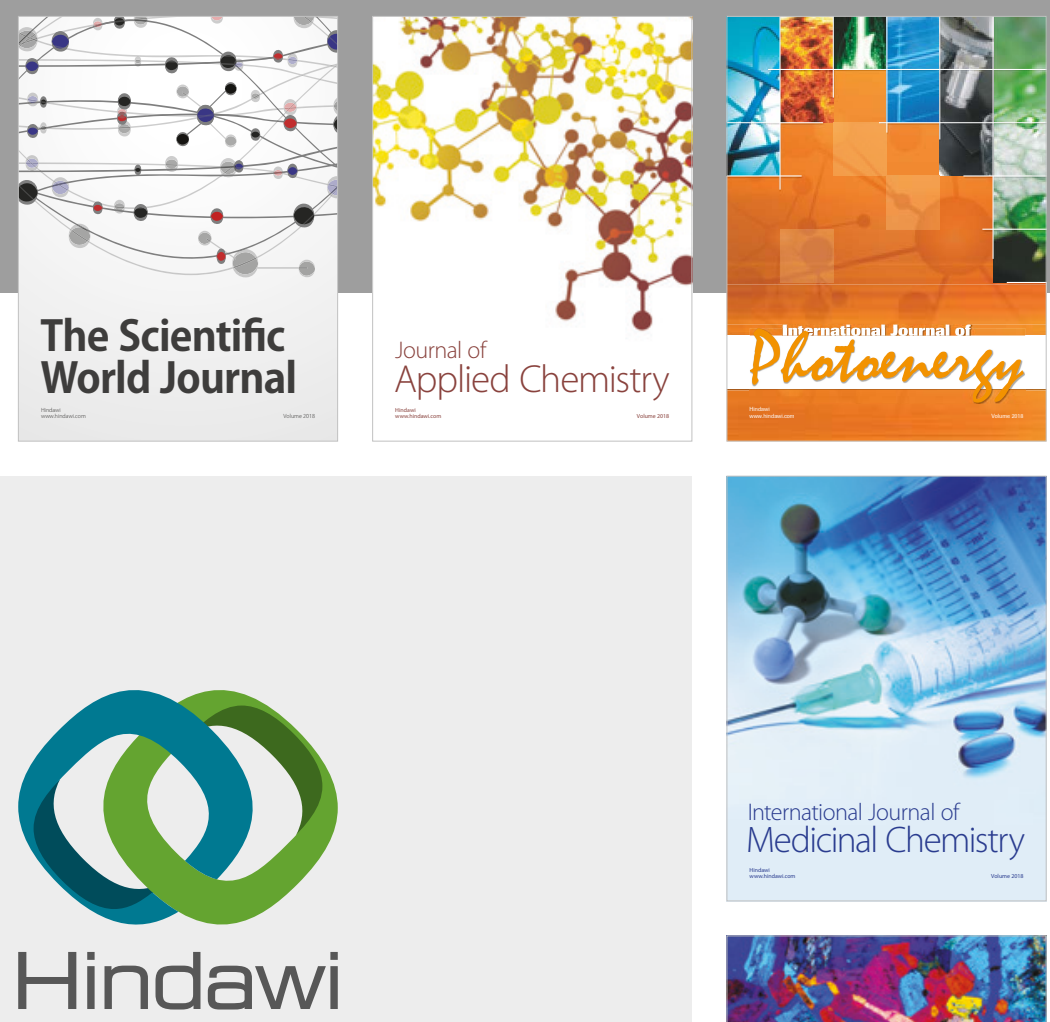

Submit your manuscripts at

www.hindawi.com
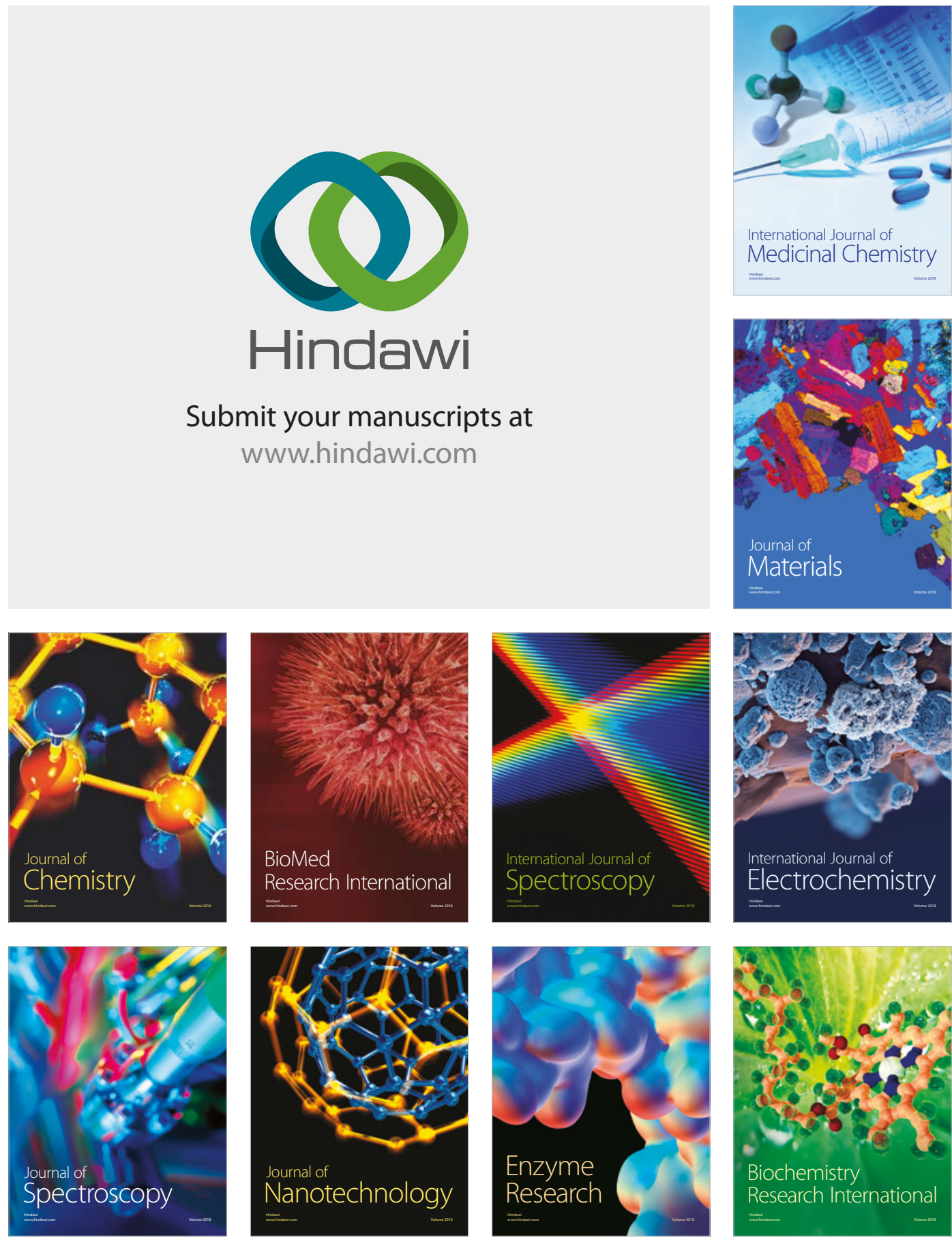
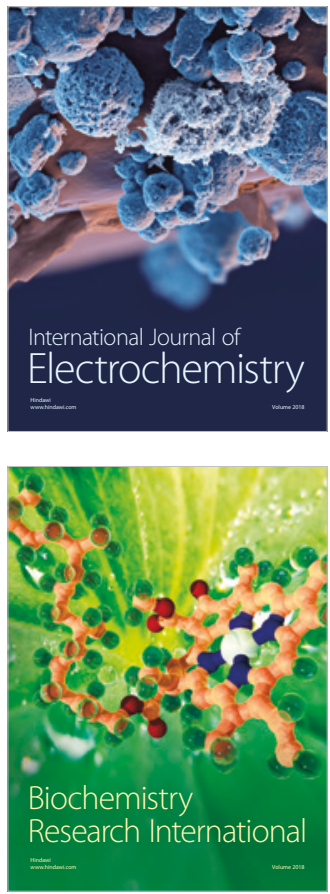